\title{
Case for diagnosis. Patch granuloma annulare*
}

\author{
Geraldo Magela Magalhães ${ }^{1,2}$ \\ Maysa Carla de Paula ${ }^{1}$
}

\author{
Cássio Ferreira Guimarães ${ }^{1}$
}

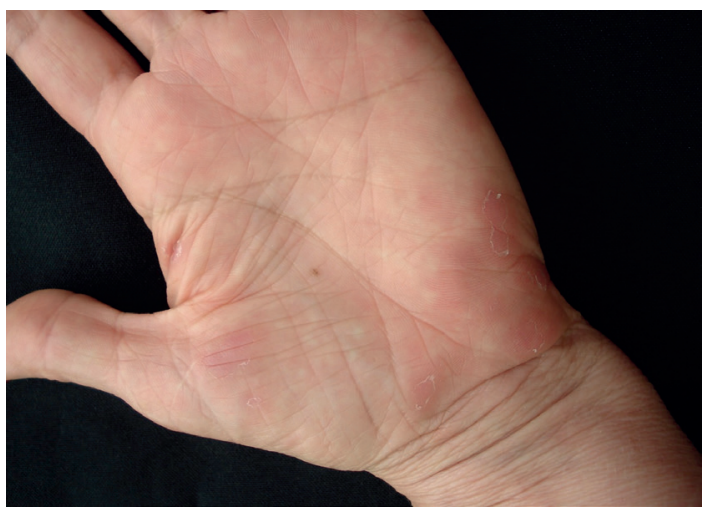

Figure 2: Slightly scaly erythematous plaques on the palm of the left hand

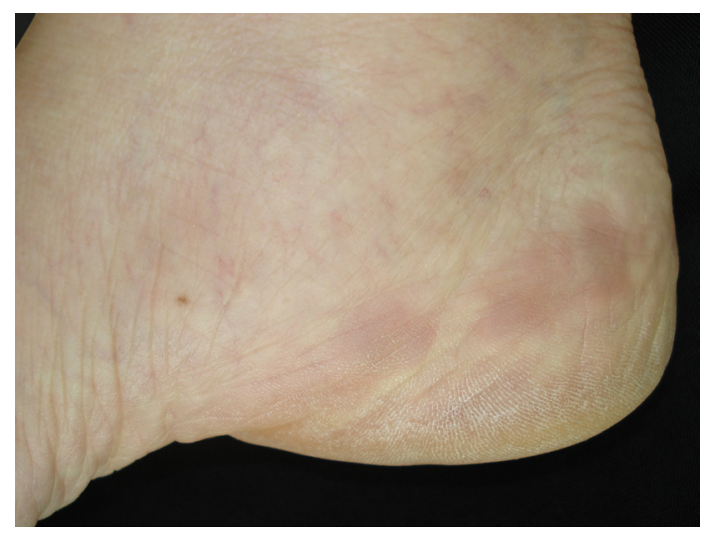

FIGURE 3: Erythematous plaques in the medial region of the right foot
FIGURE 1: Slightly scaly erythematous plaques on the palm of the right hand
A 59-year-old woman was referred to the Dermatology Service for evaluation of lesions on her hands and feet, which had appeared over the past 20 days and were asymptomatic. Her previous medical history included malignant neoplasia of the intestine, treated three years earlier, and essential arterial hypertension. She reported the use of enalapril, calcitriol and calcium carbonate, and denied allergy to any medication previously taken. A physical exam revealed slightly scaly erythematous plaques on her palms and soles (Figures 1 to 3). A skin biopsy revealed acantosis, hypergranulosis and compact ortokeratosis in the epidermis, while a lymphohistiocytic interstitial infiltrate, with an outline of epithelioid granulomas, we dermis, permeating areas of partial degeneraengulfing degenerated elastic fibers (Figure 4). There were no specific microorganisms in the sample. The lesions partially regressed after the biopsy and the remaining lesions were treated with high-potency topical corticosteroid, under occlusion, for seven days.

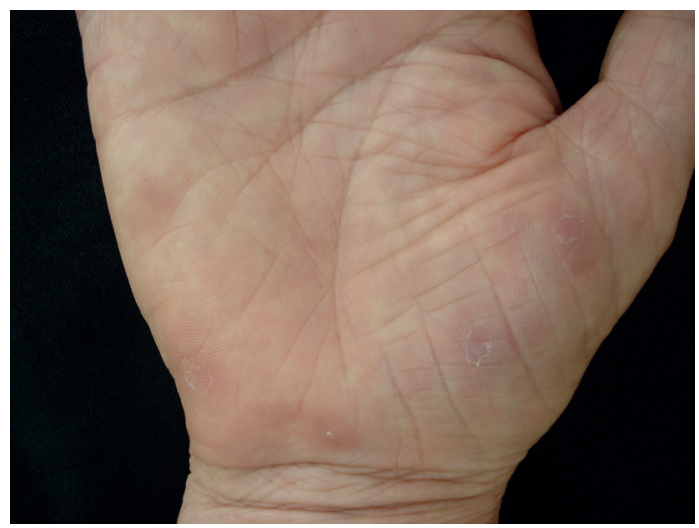

Received on 20.11.2016

Approved by the Advisory Board and accepted for publication on 24.11.2016

* Work conducted in the Dermatology Clinic, Santa Casa de Misericórdia de Belo Horizonte - Belo Horizonte, MG, Brazil.

Financial support: none.

Conflict of interest: none.

Dermatology Clinic, Santa Casa de Misericórdia de Belo Horizonte - Belo Horizonte, MG, Brazil.

Medical School, Universidade Federal de Ouro Preto (UFOP), Ouro Preto, MG, Brazil.

C2017 by Anais Brasileiros de Dermatologia 


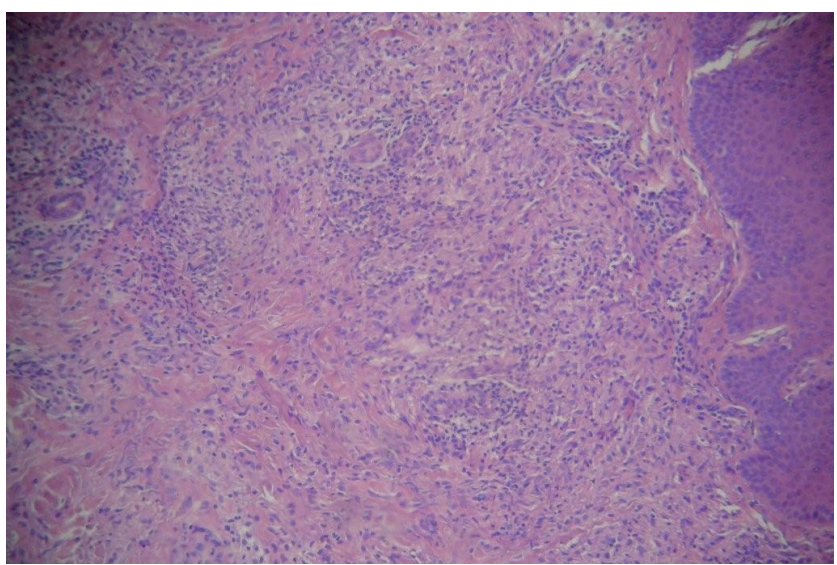

FIGURE 4: Lymphohistiocytic interstitial infiltrate with an outline of epithelioid granulomas permeating areas of partial degeneration of the collagen fibers (Hematoxylin \& eosin, x100)

\section{DISCUSSION}

Patch granuloma annulare (GA) was first reported in 1932 by Monach ${ }^{1}$ as a new macular clinical variant, which was later also named macular GA or erythematous GA. This disease is a rare variant, of unknown incidence, which is typically observed in women from 40 to 74 years of age. The clinical manifestations include large flat and erythematous patches on the trunk and limbs, which is rarely described on the palms and soles. ${ }^{2,3}$ Scalying is not a characteristic feature in the cases described in the literature. ${ }^{4}$ Clinical differential diagnoses include parapsoriasis and morphea; however, its histopathological findings are characteristic. Clinical and histological differentiation with reactive granulomatous eruptions can be challenging. ${ }^{2,5}$ The term granuloma annulare appropriately describes the classical variant of the disease, with erythematous annular plaques and granulomatous infiltrate when submitted to microscopy. Nevertheless, the disease encompasses a spectrum of clinical presentations, including the localized (most common), disseminated, subcutaneous, papular, perforating, linear and patch forms. ${ }^{2,5}$ The pathogenesis of GA, regardless of its clinical form, includes the presence of chronic vasculitis, the participation of lymphokines with the sequestration of macrophages and histiocytes in the dermis, delayed hypersensitivity reaction, defect in the chemotaxis of neutrophils and infections. ${ }^{5,6,7}$ Associations with diabetes, dyslipidemia, malignancies, infections, thyroid diseases and drugs are all reported in the literature, but they require further study in order to be confirmed. Triggers, such as contact dermatitis, tattoos, and insect bites, have also been described in the literature. ${ }^{5,8,9}$ Histologically, studies suggest that the diffuse interstitial pattern is most common in patch GA, unlike the focal palisading granuloma seen in the majority of other clinical forms. The interstitial pattern is characterized by collections of histiocytes around the collagen bands and blood vessels in the dermis. ${ }^{2}$ The resolution of GA, following many types of trauma, including biopsies, according to that observed in the present case, is a controversial phenomenon in the literature. One possible mechanism would be the reduction of the turnover of proteins from the extracellular matrix, which is naturally augmented in GA lesions, at the end of the healing process and neovascularization following the trauma. Previous case reports show that lesions with clinical and histological presentations of patch GA are more prone to post-trauma regression than those showing necrobiotic palisading granulomas. ${ }^{10} \square$

\begin{abstract}
A 59-year-old woman reported a 20-day history of slightly scaly erythematous infiltrated patches on her palms and soles with a histopathological result which was consistent with interstitial-pattern granuloma annulare, clinically classified as patch granuloma annulare. This is a rare clinical variant of granuloma annulare, with an unknown incidence and characteristic clinical and histopathological features. The patient evolved with a complete remission of the lesions after biopsy and the use of high-potency topical corticosteroid.
\end{abstract}

Keywords: Differential diagnosis; Granuloma annulare; Pathology

\section{REFERENCES}

1. Monash S. Granuloma annulare disseminatum: report of two cases. Arch Derm. 1932;25:122-31.

2. Mutasim DF, Bridges AG. Patch granuloma annulare: clinicopathologic study of 6 patients. J Am Acad Dermatol. 2000;42:417-21.

3. Stewart LR, George S, Hamacher KL, Hsu S. Granuloma annulare of the palms. Dermatol Online J. 2011;17:7.

4. Victor FC, Mengden S. Granuloma annulare, patch type. Dermatol Online J. 2008;14:21.

5. Piette EW, Rosenbach M. Granuloma annulare: clinical and histologic variants, epidemiology, and genetics. J Am Acad Dermatol. 2016;75:457-65.

6. Dahl MV, Ullman S, Goltz RW. Vasculitis in granuloma annulare: histopathology and direct immunofluorescence. Arch Dermatol. 1977;113:463-7.

7. Mempel M, Musette P, Flageul B, Schnopp C, Remling R, Gachelin G, et al. T-cell receptor repertoire and cytokine pattern in granuloma annulare: defining a particular type of cutaneous granulomatous inflammation. J Invest Dermatol. 2002;118:957-66.
8. Ragunatha S, Anitha B, Inamadar AC, Palit A, Devarmani SS. Cutaneous disorders in 500 diabetic patients attending diabetic clinic. Indian J Dermatol. 2011;56:160-4. Wu W, Robinson-Bostom L, Kokkotou E, Jung HY, Kroumpouzos G. Dyslipidemia in granuloma annulare: a case-control study. Arch Dermatol. 2012;148:1131-6.

10. Levin NA, Patterson JW, Yao LL, Wilson BB. Resolution of patch-type granuloma annulare lesions after biopsy. J Am Acad Dermatol. 2002;46:426-9.

How to cite this article: Magalhães GM, Guimarães CF, Paula MC. Case for diagnosis. Patch granuloma annulare. An Bras Dermatol. 2017;92(3):419-20. 